\title{
The Effects of Neurosculpting® Meditation on Stress, Anxiety and Sleep on one group of college students.
}

Kathleen Kelley

Quinnipiac University, 275 Mt. Carmel Ave., MNH 275K, Hamden, CT 06518, United States.

Corresponding Author: Kathleen Kelley. Quinnipiac University, 275 Mt. Carmel Ave., MNH 275K, Hamden, CT 06518, United States.

Received date: September 01, 2021; Accepted date: September 17, 2021; Published date: September 27, 2021

Citation: Kathleen Kelley. (2021) Effects of Neurosculpting® Meditation on Stress, Anxiety and Sleep on one group of college students. Clinical Research and Clinical Trials. 4(3); DOI: 10.31579/2693-4779/061

Copyright: (c) 2021 Kathleen Kelley, This is an open access article distributed under the Creative Commons Attribution License, which permits unrestricted use, distribution, and reproduction in any medium, provided the original work is properly cited.

\begin{abstract}
Objective: The purpose of this study was to examine if 11 weeks of Neurosculpting® meditation improved sleep and other variables in college aged students.

Participants: Fifteen undergraduate students.

Methods: Subjects were evaluated at the beginning and end of the semester using two tools: The Pittsburg Sleep Quality Index and the Depression Anxiety Stress Scale. During each session, heart rate and heart rate variability were measured using a smartphone app, fingertip sensor, and HRV monitor. Subjects received 60 minutes of Neurosculpting ${ }^{\circledR}$ Meditation, one time per week, for 11 weeks.

Results: The average score of both the DASS and PSQI decreased $(p=.54)$ and $(p=.08)$ respectively. Within each session, average HR decreased and average HRV increased. However, neither variable showed significant changes from the beginning to the end of the semester.

Conclusions: This study demonstrated that consistent Neurosculpting® meditation sessions may improve heart rate variability and sleep in college aged students.

Key Words: meditation; stress; anxiety; sleep; college students
\end{abstract}

\section{Introduction}

The results from the American College Health Association-National College Health Assessment (2019) indicate that the top three factors that negatively impact college student success are stress $(31.8 \%)$, anxiety $(23.2 \%)$ and sleep difficulties $(20.7 \%)$. Additionally, $9.5-31.3 \%$ of college students report suffering from depression, $5.1 \%$ report suffering from chronic illness (eg: cancer, diabetes) and 7\% report being diagnosed with ADHD. Students, however, may be hesitant to seek professional help for these problems due to negative attitudes toward mental health care [3]. Therefore, campuses around the nation are turning to alternative means to provide students with relief from stress and anxiety $[17,8]$.

Meditation has been shown to reduce anxiety and depression [15, 29] activate many areas of the brain as shown by fMRI [28, 30, 32, 22], reduce baseline cortisol levels [29] and increase attention [31]. Since college students suffer from a variety of problems and may be reluctant to seek formal mental health care, a weekly meditation class may improve sleep, and reduce anxiety and stress.

\section{Definition and Description of Meditation}

A search for synonyms of the word meditation using MSWORD (Version 14.1.0) reveals words such as "thought, consideration, deliberation, contemplation, reflection, introspection and concentration." An actual meditation practice could encompass all of these words... or none. Ancient Buddhists define meditation as the primary tool we use to see the world and mind clearly [12]. Smalley and Winston (2010) define meditation as: "Mindful awareness.....an idea that implies an awareness of self and a capacity to reflect..." (p. 1). Other authors define meditation as "A form of mental training that aims to improve an individual's core psychological capacities..." [30]. Mindfulness meditation is defined as an ability to regulate the attention. "A non-judgmental attention to present moment experiences." [31]. Ludwig and Kabat-Zin (2008) comment that "mindfulness" is a "Meditation practice that cultivates present moment awareness" (p. 1351). Although themes emerge when evaluating the definitions of meditation, many practices and opinions exist. A variety of practices exist to compliment the wide array of definitions.

Popular practices include: Mindfulness Based Stress Reduction (MBSR), Mindfulness Based Cognitive Therapy (MBCT), T'ai Chi, Sudarshan Kriya Yoga (SKY) and Yoga (Jain et al 2015, Kabat-Zinn 2019). Each form has its own signature practices and potential benefits. MBSR was 
founded by Jon Kabat-Zinn at the University of Massachusetts Medical School in 1979 [16] and is the most studied form of meditation. Neurosculpting $®$ meditation is perhaps the least studied.

Neurosculpting ${ }^{\circledR}$ is a practice designed to quiet the primitive brain, activate the prefrontal cortex and ultimately use the entire, brain right and left to engage the practitioner [34]. Initially, students begin by learning the 5-step Neurosculpting $®$ meditation process and practicing meditation. This practice may be useful for college students struggling with stress, anxiety, depression and sleeplessness. However, no studies of the effect a Neurosculpting ${ }^{\circledR}$ practice on college students have been performed. To that end, this study was performed to assess the impact of Neurosculpting® on stress, anxiety, depression, sleep and heart rate variability of one group of college students. The authors expect that Neursculpting ${ }^{\circledR}$ will reduce stress, anxiety and depression and potentially improve sleep and heart rate variability.

\section{Methods}

At the beginning of the Fall Semester, 2017, all students enrolled in the one credit course FLW 103: Introduction to Meditation, were asked to participate in the study. All students were assured that participation (or not) in no way would impact either their grade or their ability to succeed in the class. The study was approved by the XXX University IRB prior to the beginning of class in the fall of 2017.

\section{Subjects}

Twenty-nine students enrolled in FLW 103 and fifteen students agreed to participate in the study ( 9 females and 6 males). See Table 1 for full demographics. Inclusion criteria included that students were enrolled in the class and willing to be study participants. No students were excluded from the study. However, if students choose not to participate in the study portion of the class, they continued to participate in FLW 103 with no penalty or deviations from the coursework.

\section{Procedures}

On day one of class, all students were informed of the potential to participate in the study. The study was thoroughly described and students had the opportunity to ask questions of the faculty member. Students who chose to participate remained after class for approximately 30 minutes to sign the informed consent and fill out the two questionnaires: The Pittsburg Sleep Quality Index (PSQI) and the Depression Anxiety Stress Scale (DASS). Those who agreed to participate were also given a $\$ 10$ iTunes card to download the ithlete $\mathrm{TM}^{\mathrm{TM}}$ app onto their personal smartphones. This application uses a finger sensor attached to a smart phone to determine HR and HRV.

During each meditation session, heart rate (HR) and heart rate variability (HRV) were measured pre- and post-meditation. Subjects received 60 minutes of Neurosculpting® Meditation, one time per week, for 11 consecutive weeks. Sessions were conducted every Monday morning during the regular class time in the same classroom each week. At the end of each class, students transcribed HR and HRV data onto a log which was then given to the instructor. The meditation program was taught by a Certified Neurosculpting® Meditation Teacher.

Finger sensors were provided by the faculty member each session. The last 30 minutes of the final class were used for the participants to retake the PSQI and the DASS. The faculty member who taught Neurosculpting ${ }^{\circledR}$ was blind to the results of all Pre- and Post-tests (DASS, PSQI, HR and HRV). All tests were kept in a locked cabinet it a locked office.

\section{Measures}

\section{Pittsburg Sleep Quality Index (PSQI)}

The Pittsburg Sleep Quality Index (PSQI) is a self-report questionnaire designed to measure both the quality of a participants' sleep and possible disturbances to sleep. The questionnaire contains ten main questions with sub-questions under numbers five and ten. The questionnaire is scored based upon seven components. Researchers can derive a "global score" from adding all seven sections together [5]. Test-retest reliability is reported as .87 and validity analysis shows that a score of greater than 5 correlates with sleep disturbances in subjects with established insomnia [2].

Previous studies [5, 23] using the PSQI have indicated the importance of studying the sleep quality of individuals. Sleep disturbances can be a symptom of sleep and/or medical disorders and may correlate with mortality. The PSQI allows researchers to examine the effects of weekly meditation on sleep by quantifying both the changes in sleep and sleep quality $[5,23]$.

\section{Depression Anxiety Stress Scale (DASS)}

The Depression Anxiety Stress Scale (DASS) is a 42-item self-report instrument designed to measure depression, anxiety and stress [18] The DASS contains a set of three self-report scales, each with 14 items. This scale discriminates the differences among the emotional states of depression, anxiety and stress (Overview of DASS, 2018). Test re-test reliability of the DASS has been reported as .71 for depression, .79 for anxiety and .81 for stress [4]. This self-report measure was selected for this study to examine the effects of Neurosculpting® on depression, stress and anxiety in college students.

\section{Heart Rate Variability}

Heart rate variability (HRV) is defined as "the quantification of beat-tobeat variability in the cardiac cycle over time [13]. Different than a heartbeat, which is the number of times the heart muscle completes a period of contraction and relaxation per minute, HRV tracks the time between each heartbeat to measure both the function of the autonomic nervous system (ANS) and its ability to communicate with the heart. This measurement monitors how the heart copes with various external factors.

Previous methods of collecting HRV data involved assessing a subject's electrocardiograph (ECG). ECG collection is both cumbersome and time consuming. Current methods used to measure HRV employ the use of a finger sensor along with the ithlete ${ }^{\mathrm{TM}}$ smart phone application. This smartphone application was created to measure stress levels in athletes in an objective manner [9]. And has become a popular and reliable measurement technique to determine short term HRV measures in healthy adult subjects. Short term measurements of 60 seconds in duration have been shown to be adequate for measuring HRV in collegiate athletes (Esco \& Flatt 2014). To ensure the ithlete ${ }^{\mathrm{TM}}$ smartphone application's validity, [9] collected data comparing a conventional ECG setup with data collected using the ithlete ${ }^{\mathrm{TM}}$ application. Researchers showed that the ithlete $^{\mathrm{TM}}$ application provides a time domain of HRV index, known as root mean square of successive R-R intervals (RMSSD), which reflects parasympathetic modulation of the heart. The RMSSD is an indicator of both physical and non-physical stressors [9]. Determined that HRV values obtained via the ithlete ${ }^{\mathrm{TM}}$ smartphone application provide an accurate measure of HRV when compared to measures obtained with ECG.

\section{Overview of the Neurosculpting $®$ Program}

Neurosculpting® is a 5-step meditation process that may encourage an individual to release the grip of old patterns and entrain their brain to new and more supportive patterns, habits and behaviors. Neurosculpting ${ }^{\circledR}$ was developed as a method to support self-directed neuroplasticity of the brain. Neurosculpting $®$ employs a unique methodology by intentionally engaging the whole-brain through specific language and focused imagery. This unique methodology was developed in response to both years of 
meditation practice and study of the neuroscience of brain function by its founder, Lisa [34].

Neurosculpting® uses a unique, specific 5-step process to retrain the brain:

Step One: Down-regulates hyperactive stress activity via an engagement with the parasympathetic response.

Step Two: Enhances focused attention to support with emotional regulation.

Step Three: Increases the activity between our analytical self and our intuitive feeling self via engaging the whole brain (Right and Left sides)

Step Four: Links somatosensory, bodily sensation-based, engagement to perceptual shifts in patterns.

Step Five: Enables the user to easily identify and replicate the process in day-to-day activities

During the meditation (immediately prior to initiating the practice and immediately after), HR and HRV were monitored as described above.
Each student recorded pre-meditation and post-meditation HR and HRV on a personal log. Logs were collected by the primary investigator each week and stored in a locked cabinet in locked office.

\section{Data Analysis}

PSQI and DASS data were analyzed using a paired t-test. HR and HRV data were analyzed using a mixed model analysis with fixed effects. All data were analyzed using SPSS v24.

\section{Results}

After 11 weeks of meditation, the average score for the DASS decreased from 22.07 to $20.07(t=.626 ; p=.542$, not significant) (Figure 1$)$ and the average PSQI score decreased from 6.37 to $5.93(\mathrm{t}=1.87 ; \mathrm{p}=.08)$ (Figure 2).

After 11 weeks of meditation, overall resting heart rate showed no significant reduction. HR was noted to decrease within sessions each week $(\mathrm{p}=.00)$, from pre- to post- meditation (except Week 7, see Figure 3 ). HRV increased after each session $(p=.00)$ except weeks 6 and 9 (see Figure 4).

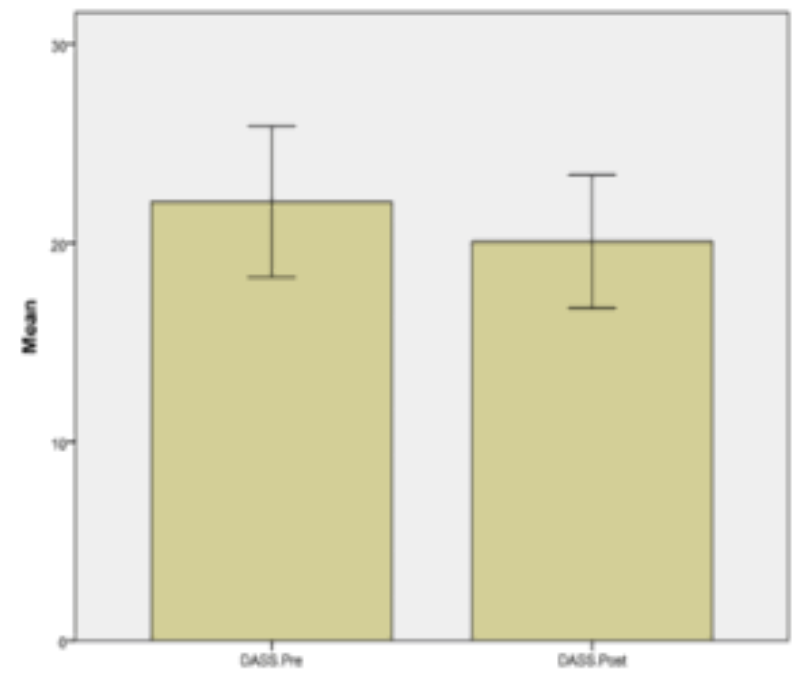

Figure 1: Average Score, DASS

The average score for the DASS decreased from 22.07 to $20.07(\mathrm{t}=.626 ; \mathrm{p}=.542)$

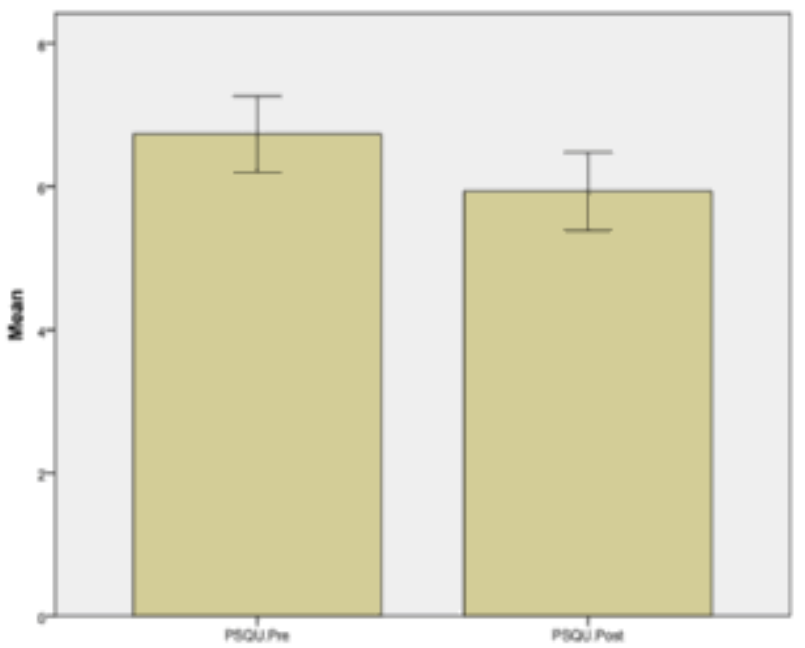

Figure 2: PSQI Pre and Post Test Data

Average PSQI score decreased from 6.37 to $5.93(\mathrm{t}=1.87 ; \mathrm{p}=.08)$ 


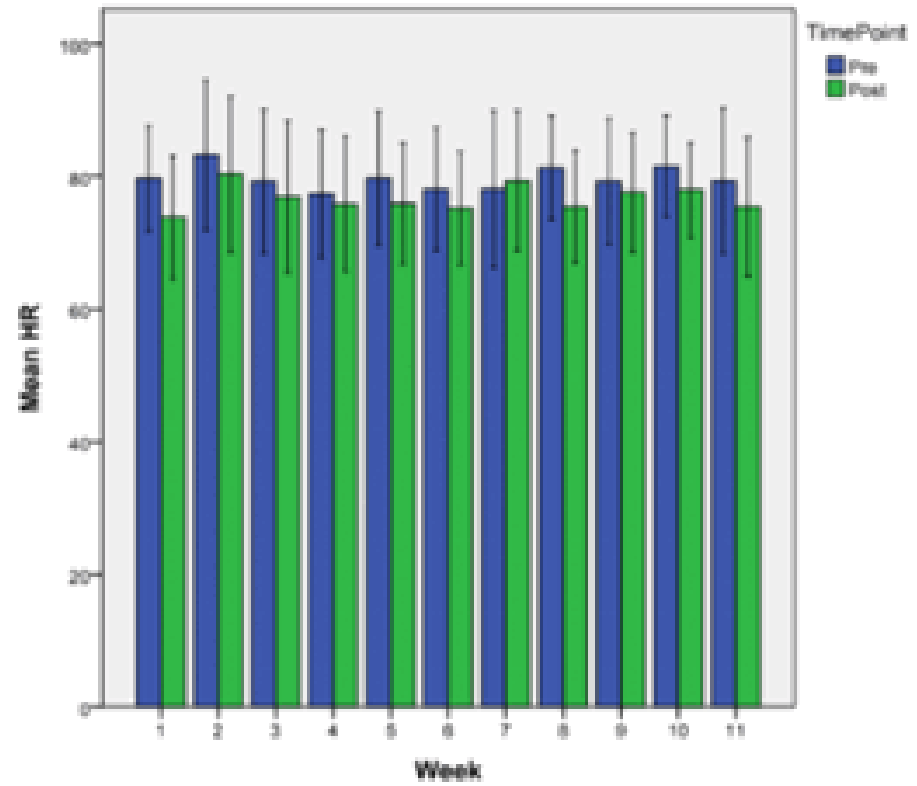

Figure 3: HR Data over time

For all weeks except week 7, mean HR reduces from pre- to post- meditation $(\mathrm{p}=.00)$

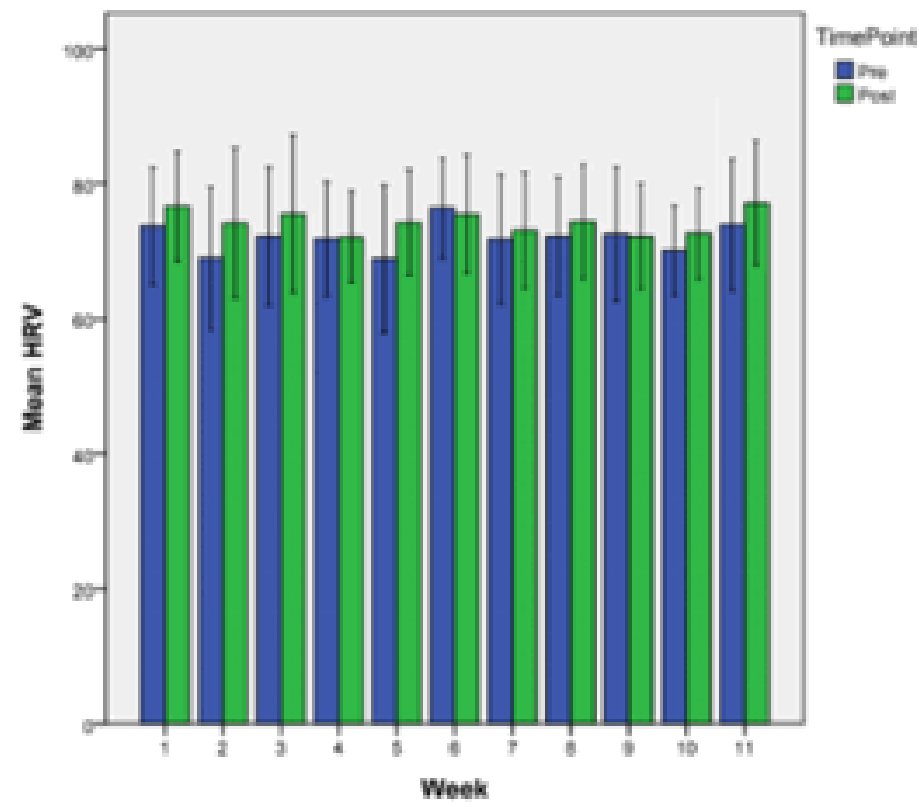

HRV increased after each session $(\mathrm{p}=.00)$

\section{Figure 4: HRV Data over time}

\section{Discussion}

This study revealed that a weekly Neurosculpting® Meditation class may improve sleep but does not appear to impact on stress, anxiety, or depression in one group of college students. Neurosculpting® Meditation also has short-term but not long-term impact on HR and HRV.

\section{Meditation and Sleep}

Over twenty percent of college students report experiencing sleep difficulties [1]. In addition, $50 \%$ of college students report daytime sleepiness [24], 70\% report getting less than eight hours of sleep per night and $60 \%$ are considered to have poor quality sleep [20]. Poor quality sleep and sleep deprivation can lead to decreased grade point averages, impaired academic performance, impaired ability to drive safely and, in a small percentage of students, suicidal thoughts [14]. Other authors have shown that meditation improves sleep $[11,10]$. In this study, $93.3 \%$ of participants exhibited poor sleep quality (as measured by the PSQI) at the pre-test. However, at post-test, this number decreased to $66.6 \%$, indicating that a weekly meditation practice may have improved sleep 
quality. These results confirm what other authors have found, that meditation may improve sleep quality $[10,35,10]$.

Given the prevalence of sleep disturbances in college students and the plethora of detrimental consequences, any program that improves sleep even a slight amount is beneficial. Further studies are needed with more subjects and more frequent meditation sessions to determine the full benefit of a Neurosculpting® Meditation practice on sleep disorders in college students.

\section{Meditation and Autonomic Regulation}

HR and HRV are indicators of autonomic regulation of the cardiovascular system [35]. Acceleration of HR is caused by sympathetic regulation and change in HRV is caused by parasympathetic regulation [6]. HRV increases in a more parasympathetic or relaxed state. Our results indicate that after each Neurosculpting ${ }^{\circledR}$ session, the sympathetic system down regulated $\mathrm{HR}$ and the parasympathetic system upregulated HRV. Therefore, a short term, but not long- term effect was seen on both HR and HRV after a meditation session. This short-term decrease in HR and increase in HRV from pre- to post- meditation session demonstrates successful inhibition of sympathetic tone and activation of parasympathetic tone [29]. The lack of significant long -term effects may be secondary to the class time constraints of 1 hour, $1 \mathrm{X}$ per week and/or the short duration of the class (11 weeks). However, the short- term changes seen indicate that the subjects' have healthy, adaptable cardiovascular systems. A long-term effect may be seen if the subjects continued Neurosculpting® meditation either at a higher weekly frequency or a longer duration.

\section{Limitations}

There were several limitations to the study. The sample group was small (15 subjects) and the majority of participants who did complete the study missed at least one class. Anecdotally, the participants who had perfect attendance had greater, but not significantly different, changes on both the DASS and PSQI, indicating dose may be an important variable that impacts success of a mediation program. Dose was also limited by the constraints of the college semester, allowing for only 11 weeks of data collection. A longer study may result in larger and more permanent behavioral changes. Finally, the sample population was a group of generally healthy, well-educated young college students and therefore may not be universally generalizable to all college students.

\section{Conclusion}

This study demonstrated that 11 weeks of a one-hour long Neurosculpting ${ }^{\circledR}$ meditation session can reduce stress and anxiety and improve sleep in college students. In addition, Neurosculpting® meditation has a positive temporary effect on resting heart rate and heart rate variability.

\section{References}

1. American College Health Association-National College Health Assessment (ACHA-NCHA), Spring 2019 Reference Group Executive Summary.

2. Backhaus J, Junghanns K, Broocks A, Riemann D, Hohagen, F. (2002). Test-retest reliability and validity of the Pittsburgh Sleep Quality Index in primary insomnia. Journal of Psychosomatic Research. 53: 737-740

3. Bohon L, Cotter K, Kravitz R, Cello P, Garcia EF. (2016). The Theory of Planned Behavior as it predicts potential intension to seek mental health services for depression among college students. J of American College Health. 64:593-603.
4. Brown TA, Chorpita, BF, Lorotitsch W, Barlow D. (1997). Psychometric Properties for the 42-item and 21-item version of the Depression, Anxiety Stress Scales (DASS) in clinical groups and a community sample. Psychological Assessment. 10: 176-181.

5. Buysse DJ, Reynolds CF, Monk TH, Berman SR, Kupfer, DJ. (1988). The Pittsburgh Sleep Quality Index: A New Instrument for Psychiatric Practice and Research. Psychiatry Research. 28:193-213

6. Camm A, Malik M, Bigger J, Breithardt G, Cerutti S, Cohen R, et al. (1996). Heart rate variability: standards of measurement, physiological interpretation and clinical use. Task Force of the European Society of Cardiology and the North American Society of Pacing and Electrophysiology. Circulation. 93: 1043-1065.

7. Esco MR, Flatt AA. (2014). Ultra-short-term heart rate variability indexes at rest and post-exercise in athletes: evaluating the agreement with accepted recommendations. J Sports Sci Med. 13: 535-541.

8. Ewing B, Ryan M, Zarco E. (2007). A campus wellness program: Accepting the challenge. The Journal of the New York State Nursing Association. 38: 13-16.

9. Flatt AA, Esco MR. (2013). Validity of the ithleteTM smart phone application for determining

10. Gong H, Ni CS, Lu YZ, Zhang Y, Su WJ, Lian YJ et al. (2016). Mindfulness meditation for insomnia: A meta-analysis of randomized control trials. J of Psychosomatic Research. 89: 16.

11. Gross C, Kreitzer MH, Reilly-Spong, Wall W, Winbush NY, Patterson R et al. (2011). Mindfulness-based stress reduction versus pharmacotherapy for chronic primary insomnia: a randomized controlled trial. Explore-J Sci Heal. 7:76-87.

12. Hanson R. (2010). Mendius R.Buddha's Brain, Oakland, CA: New Harbinger Publications

13. Heathers J. (2013). Smartphone-enabled pulse rate variability: an alternative methodology for the collection of heart rate variability in psychophysiological research. Int J Psychophysiol. 89: 297-304.

14. Hershner S, Chervin R. (2014). Causes and consequences of sleepiness among college students. Nature and Science of Sleep. 6:73-84.

15. Jain F, Walsh R, Eisendrath S, Christensen S, Cahn R. (2015). Critical Analysis of the Efficacy of Meditation Therapies for Acute and Subacute Phase Treatment of Depressive Disorders: A Systematic Review, Psychosomatics. 56:140-152.

16. Kabat-Zinn, J. Center of mindfulness in medicine, healthcare and society. Meet our Faculty.

17. Klainberg M, Ewing B, Ryan M. (2010). Reducing stress on a college campus. The Journal of the New York State Nursing Association, Fall/Winter. 4 -10.

18. Lovibond SH, Lovibond PF. (1995). Manual for the Depression Anxiety Stress Scales, 2nd ed. Sydney: Psychology Foundation.

19. Lovibond PF, Lovibond SH. (1995). The structure of negative emotional states: Comparison of the Depression Anxiety Stress Scales (DASS) with the Beck Depression and Anxiety Inventories. Behaviour Research and Therapy. 33: 335-343.

20. Lund H, Reider B, Whiting A, Prichard J. (2010). Sleep Patterns and Predictors of Disturbed Sleep in a Large Population of College Students. Journal of Adolescent Health. 46: 124-132.

21. Ludwig M, Kabat-Zinn, J. (2008). Mindfulness in Medicine, JAMA. 300:1350-1352.

22. Lutz J, Bruhl A, Scheerer H, Jancke L, Herwig 2016. Neural correlates of mindful self-awareness in mindfulness meditators 
and meditation-naïve subjects revisited. Biological Psychology. 119: 21-30.

23. Mollayeva T, Thurairajah P, Burton K, Mollayeva S, Shapiro CM, Colantonio A. (2015). The Pittsburgh sleep quality index as a screening tool for sleep dysfunction in clinical and nonclinical samples: A systematic review and meta-analysis. Sleep medicine reviews. 25: 52-73.

24. Oginaka H, Pokorski, J. (2006). Fatigue and Mood Correlates of Sleep Length in Three Age-Social Groups: School Children, Students, and Employees. Chronobiology International: The Journal of Biological and Medical Rhythm Research. 23:13171328.

25. Overview of the DASS and its uses.

26. Rickwood D, Deane F, Wilson C, Ciarrochi, J. (2005). Young people's help-seeking for mental health problems. Australian eJournal for the Advancement of Mental Health, 4: 218-251.

27. Smalley S, Winston D. (2010). Fully Present: The Science, Art, and Practice of Mindfulness. Philadelphia: De Capo Press

28. Sperduti M, Martinelli P, Piolina, P. (2012). A neurocognitive model of meditation based on activation likelihood estimation (ALE) meta-analysis. Consciousness and Cognition. 21: 269276.
29. Tang Y, Wang J, Fan Y, Feng S, Lu Q, Yu Q, et al. (2007). Short-term meditation training improves attention and selfregulation. ProcNatl Acad Sci USA. 04: 17152-17156.

30. Tang YY, Holzel B, Posner M. (2015). The neuroscience of mindfulness meditation. Nature Reviews. 16: 313-325.

31. Tang YY, Leve L. (2016). A translational neuroscience perspective on mindfulness meditation as a prevention strategy. TBM. 6: 63-72.

32. Taren A, Gianaros P, Greco C, Lindsay E, Fairgrieve A, Brown $\mathrm{K}$ et al. (2015). Mindfulness meditation training alters stressrelated amygdala resting state functional connectivity: A randomized control trial, Social Cognitive and Affective Neuroscience. 1758-1768.

33. Vanheusden $\mathrm{K}$, Mulder $\mathrm{C}$, van der Ende J, van Lenthe $\mathrm{F}$, Mackenbach J, Verhulst, F. (2008) Young adults face major barriers to seeking help from mental health services. Patient Educ Couns. 73:97-104.

34. Wimberger L. (2016). Neurosculpting: A Whole-Brain Approach to Heal Trauma, Rewrite Limiting Beliefs and Find Wholeness. Boulder, CO: Sounds True

35. Winbush N, Gross C, Kreitzer, M. (2007). The effects of mindfulness based stress reduction on sleep disturbance: a systematic review. Explore (NY). 3:585-591.
This work is licensed under Creative Commons Attribution 4.0 License

To Submit Your Article Click Here: Submit Manuscript

DOI: $10.31579 / 2693-4779 / 061$

\author{
Ready to submit your research? Choose Auctores and benefit from: \\ $>$ fast, convenient online submission \\ $>$ rigorous peer review by experienced research in your field \\ $>$ rapid publication on acceptance \\ $>$ authors retain copyrights \\ $>$ unique DOI for all articles \\ $>$ immediate, unrestricted online access
}

At Auctores, research is always in progress.

Learn more https://www.auctoresonline.org/journals/clinical-research-andclinical-trials 